\title{
FLIGHT TEST VALIDATION OF OPTIMAL INPUT DESIGN AND COMPARISON TO CONVENTIONAL INPUTS
}

\author{
Eugene A. Morelli* \\ NASA Langley Research Center \\ Hampton, Virginia USA 23681-0001
}

\begin{abstract}
$\underline{\text { Abstract }}$
A technique for designing optimal inputs for aerodynamic parameter estimation was flight tested on the F-18 High Angle of Attack Research Vehicle (HARV). Model parameter accuracies calculated from flight test data were compared on an equal basis for optimal input designs and conventional inputs at the same flight condition. In spite of errors in the a priori input design models and distortions of the input form by the feedback control system, the optimal inputs increased estimated parameter accuracies compared to conventional 3-2-1-1 and doublet inputs. In addition, the tests using optimal input designs demonstrated enhanced design flexibility, allowing the optimal input design technique to use a larger input amplitude to achieve further increases in estimated parameter accuracy without departing from the desired flight test condition. This work validated the analysis used to develop the optimal input designs, and demonstrated the feasibility and practical utility of the optimal input design technique.
\end{abstract}

\section{Nomenclature}

$\mathbf{A}, \mathbf{B}, \mathbf{C}, \mathbf{D}$ system matrices

$a_{y}, a_{z} \quad$ linear accelerations, g's

$g \quad$ acceleration due to gravity, $\mathrm{ft} / \mathrm{sec}^{2}$

$J \quad$ cost function

M information matrix

$N \quad$ total number of sample times

$p, q, r \quad$ body axis angular velocities, $\mathrm{rad} / \mathrm{sec}$

$\mathbf{R} \quad$ discrete noise covariance matrix

$\mathbf{S}(i) \quad$ output sensitivity matrix at time $i \Delta t$

$T \quad$ maneuver duration, sec

u $\quad n_{i}$-dimensional control vector

$V \quad$ airspeed, $\mathrm{ft} / \mathrm{sec}$

$\mathbf{x} \quad n_{s}$-dimensional state vector

\begin{tabular}{|c|c|}
\hline $\begin{array}{l}\mathbf{y} \\
\mathbf{y}(i)\end{array}$ & $\begin{array}{l}n_{o} \text {-dimensional output vector } \\
\text { output vector at time } i \Delta t\end{array}$ \\
\hline$\alpha$ & angle of attack, rad \\
\hline$\beta$ & sideslip angle, rad \\
\hline$\delta_{i j}$ & Kronecker delta \\
\hline$\Delta t$ & sampling interval, sec \\
\hline$\phi$ & roll angle, rad \\
\hline$\delta_{a}$ & aileron deflection, rad \\
\hline$\delta_{a_{s y m}}$ & symmetric aileron deflection, rad \\
\hline$\delta_{f}$ & trailing edge flap deflection, rad \\
\hline$\delta_{r}$ & rudder deflection, rad \\
\hline$\delta_{s}$ & stabilator deflection, rad \\
\hline$\mu_{j}$ & $j^{\text {th }}$ input amplitude constraint \\
\hline $\mathbf{v}(i)$ & $i^{\text {th }}$ discrete measurement noise vector \\
\hline$\Theta$ & pitch angle, rad \\
\hline $\boldsymbol{\theta}$ & $n_{p}$-dimensional parameter vector \\
\hline$\theta_{j}$ & $j^{\text {th }}$ model parameter \\
\hline$\sigma_{j}$ & Cramér-Rao bound for the $j$ th parameter \\
\hline$\xi_{k}$ & $k^{\text {th }}$ output amplitude constraint \\
\hline \multicolumn{2}{|c|}{$\underline{\text { subscripts }}$} \\
\hline$o$ & average value \\
\hline$m$ & measured \\
\hline \multicolumn{2}{|c|}{ superscripts } \\
\hline$T$ & transpose \\
\hline-1 & matrix inverse \\
\hline
\end{tabular}

\section{Introduction}

Experiment design is important for identifying high fidelity mathematical models of modern aircraft from flight test data. The flight test maneuver (equivalently, the flight test input) has a major impact on the quality of the data for modeling purposes. A good experiment design must account for practical constraints during the flight test, while maximizing effectiveness of expensive flight test time. 
The overall goal is to design an experiment that produces data from which model parameters can be estimated accurately. This translates into exciting the system modes so that the sensitivities of the model outputs to the parameters are high and correlations among the parameters are low. Frequency sweep inputs ${ }^{1}$ can be used to do this, requiring little more than knowledge of the frequency range of interest for the modeling. This technique is restricted to moving a single input at a time, so that off-axis responses or coupled motions are generally not well modeled from frequency sweep data. Frequency sweeps also require relatively long maneuver times (i.e., 1-2 minutes) to run through the frequency range of interest. Low frequency components of the frequency sweep contribute to long maneuver times, and also increase the tendency for the aircraft to depart from the desired flight test condition. For high performance aircraft, limited flight test time, multiple control effectors, and flight conditions such as high angle of attack make the frequency sweep approach difficult to use and expensive.

An alternate approach is to take advantage of a priori knowledge about the dynamics of the aircraft to focus the input energy at frequencies near the system modes. An a priori model can be assembled using wind tunnel aerodynamic data and knowledge of rigid body dynamics. This permits the design of short flight test maneuvers with high information content that can be analyzed using maximum likelihood parameter estimation in the time domain ${ }^{2,3}$. A paradox occurs here, in that very good inputs will be designed when the a priori model is very good; however, in this case the experiment is less needed. Obviously, the input design technique must be robust to errors in the a priori model.

Designing an input for accurate model parameter estimation requires rich excitation of the system, which is frequently at odds with various practical constraints. One such practical constraint is the requirement that output amplitude excursions (e.g., in angle of attack or sideslip angle) about the flight test condition be limited in order to assure the validity of an assumed model structure. Input amplitudes must be constrained for the same reasons, and to avoid nonlinearities such as mechanical stops and rate limiting when the model is linear.

Designing an input that excites the aircraft dynamic response as much as possible when modal frequencies are imperfectly known, while simultaneously satisfying practical constraints, is a difficult problem. Several researchers have studied the problem of finding optimal inputs for aircraft parameter estimation ${ }^{4-13}$. The most serious obstacles to using the results of these studies in flight have been practical implementation issues. These include unrealizable optimal input forms, and failure to account for closed-loop control, actuator dynamics or constraints on input and output amplitudes. Computationally, the difficulties have been selection of an appropriate optimality criterion, inadequate numerical optimization techniques for finding global optimal solutions, and difficulties associated with multiple input design.

Recent research ${ }^{14-16}$ has produced an optimal input design technique which addresses the above issues. The technique generates square wave inputs which are globally optimal in the sense that information content in the data is maximized for a fixed flight test time, or, alternatively, specified parameter accuracy goals are achieved in minimum flight test time.

The optimal input design technique has been shown to be theoretically sound ${ }^{14,15}$, has been validated in flight for aerodynamic model parameter estimation experiments using pilot implementation, including demonstrated higher parameter accuracies compared to compound doublet inputs ${ }^{16}$, has been used successfully to specify flight test maneuvers for closed loop flying qualities model identification at high angles of attack ${ }^{17}$, and has compared favorably to other techniques in the literature for a standard test problem ${ }^{18}$. In the latter reference, the global optimal square wave input produced the lowest value of the sum of estimated parameter variances, even though the maneuver time allotted for the optimal square wave input design was the smallest of any of the techniques studied (see Table 3 of Ref. [18], p. 281). This fact, though not pointed out by the authors of Ref. [18], demonstrates the effectiveness of the optimal input design technique.

The purpose of this work was to test the optimal input design technique in flight using a computerized system to implement the optimal inputs, and to compare flight test results from the optimal inputs to results from conventional 3-2-1-1 and doublet inputs similarly implemented. The 3-2-1-1 input form has been shown to be very effective for aircraft parameter estimation in previous flight test investigations ${ }^{11,12}$.

The next section outlines the theory involved in the optimal input design technique. Next, the F-18 High Alpha Research Vehicle (HARV) test aircraft and some details of the flight test procedure are described. Following this, the results from the flight tests are presented and discussed.

\section{Theoretical Development}

Airplane dynamics can be described by the following linear model equations:

$$
\begin{gathered}
\dot{\mathbf{x}}(t)=\mathbf{A} \mathbf{x}(t)+\mathbf{B} \mathbf{u}(t) \\
\mathbf{x}(0)=\mathbf{0} \\
\mathbf{y}(t)=\mathbf{C} \mathbf{x}(t)+\mathbf{D} \mathbf{u}(t) \\
\mathbf{y}_{m}(i)=\mathbf{y}(i)+\mathbf{v}(i) \quad i=1,2, \ldots, N
\end{gathered}
$$


Elements of the state vector $\mathbf{x}$, control vector $\mathbf{u}$, and output vector $\mathbf{y}$ are perturbation quantities. The measurement noise $\mathbf{v}(i)$ is assumed Gaussian with

$$
\mathrm{E}\{\mathbf{v}(i)\}=\mathbf{0} \quad \text { and } \quad \mathrm{E}\left\{\mathbf{v}(i) \mathbf{v}^{T}(j)\right\}=\mathbf{R} \delta_{i j}
$$

Nonlinear models can be used in Eqs. (1) and (3) without any modification in the following development of the optimal input design procedure. Linear models are used in Eqs. (1) and (3) because of the common practice of estimating stability and control derivatives from flight test data, and for consistency with the data analysis to be given later.

The input quantities were control surface deflections, with output quantities from air data $(\alpha, \beta)$, body axis angular velocities $(p, q, r)$, Euler angles $(\phi)$, and translational accelerations $\left(a_{y}, a_{z}\right)$. Longitudinal and lateral cases were treated separately, with the linear model structure shown above resulting from the usual small perturbation assumptions ${ }^{2}$.

Constraints arising from practical flight test considerations were imposed on all input amplitudes and selected output amplitudes. Control surface amplitudes are limited by mechanical stops, flight control software limiters, or linear control effectiveness. Selected output amplitudes must be limited to avoid departure from the desired flight test condition and to ensure validity of the assumed linear model structure. In addition, constraints may be required on aircraft attitude angles for flight test operational considerations, such as flight safety and maintaining line of sight from the downlink antenna aboard the aircraft to the ground station. The constraints were specified by

$$
\begin{gathered}
\left|u_{j}(t)\right| \leq \mu_{j} \quad \forall t ; \quad j=1,2, \ldots, n_{i} \\
\left|y_{k}(t)\right| \leq \xi_{k} \quad \forall t ; \quad k \in\left(1,2, \ldots, n_{o}\right)
\end{gathered}
$$

where $\mu_{j}$ and $\xi_{k}$ are positive constants.

When estimating model parameter values from measured data, the minimum achievable parameter standard errors using an asymptotically unbiased and efficient estimator (such as maximum likelihood) are called the Cramér-Rao lower bounds $2,3,14$. These quantities are a function of the excitation of the system and the noise levels, and collectively measure the information content in the data. For a fixed instrumentation system, the Cramér-Rao lower bounds are influenced by the excitation of the system, which is determined by the choice of the input. The choice of input implicitly includes the length of the maneuver.
The Cramér-Rao lower bounds for the parameter standard errors are given by the square root of the diagonal elements of the dispersion matrix $\mathbf{D}^{2,3,14}$. The dispersion matrix is defined as the inverse of the information matrix $\mathbf{M}$, the latter being a measure of the information content of the data from an experiment. The expressions for these matrices are

$$
\begin{gathered}
\mathbf{M}=\sum_{i=1}^{N} \mathbf{S}(i)^{T} \mathbf{R}^{-1} \mathbf{S}(i) \\
\mathbf{D}=\mathbf{M}^{-1}
\end{gathered}
$$

where $\mathbf{S}(i)$ is the matrix of output sensitivities to the parameters,

$$
\mathbf{S}(i)=\left.\frac{\partial \mathbf{y}(i)}{\partial \boldsymbol{\theta}}\right|_{\boldsymbol{\theta}=\hat{\boldsymbol{\theta}}}
$$

and $\hat{\boldsymbol{\theta}}$ denotes the parameter vector estimate. The output sensitivities for the $j^{\text {th }}$ parameter appear as the $j^{\text {th }}$ column of the sensitivity matrix, and are computed from

$$
\begin{gathered}
\frac{d}{d t}\left[\frac{\partial \mathbf{x}}{\partial \theta_{j}}\right]=\mathbf{A} \frac{\partial \mathbf{x}}{\partial \theta_{j}}+\frac{\partial \mathbf{A}}{\partial \theta_{j}} \mathbf{x}+\frac{\partial \mathbf{B}}{\partial \theta_{j}} \mathbf{u} \\
\frac{\partial \mathbf{x}}{\partial \theta_{j}}(0)=\mathbf{0} \\
\frac{\partial \mathbf{y}}{\partial \theta_{j}}=\mathbf{C} \frac{\partial \mathbf{x}}{\partial \theta_{j}}+\frac{\partial \mathbf{C}}{\partial \theta_{j}} \mathbf{x}+\frac{\partial \mathbf{D}}{\partial \theta_{j}} \mathbf{u}
\end{gathered}
$$

for $j=1,2, \ldots, n_{p}$. Eqs. (11)-(13) follow from differentiating Eqs. (1)-(3) with respect to $\theta_{j}$, combined with the assumed analyticity of $\mathbf{x}$. The output sensitivities $\mathbf{S}(i)$ can also be computed using finite differences.

From Eqs. (8)-(13), it is clear that the information matrix elements (and therefore the Cramér-Rao bounds) depend on the input through the sensitivity equations (11)-(13). The input $\mathbf{u}$ influences the sensitivities both directly as a forcing function in the sensitivity equations and indirectly as an influence on the states, which also force the sensitivity equations. The dependence of the Cramér-Rao bounds on the input is nonlinear in the input amplitude, regardless of whether or not the system equations (1) and (3) are linear, because of the nonlinear character of Eqs. (8) and (9).

Equation (8) is a discrete approximation to a time integral over the maneuver duration $T$. Therefore, when comparing the effectiveness of various input designs 
using some function of the dispersion matrix $\mathbf{D}$ as the criterion for comparison, the input designs being compared should have the same maneuver duration and, in light of the last paragraph, also the same allowable maximum input amplitude. This approach contrasts with comparisons presented in previous works $11,12,18$, which were based on constant input energy. If only constant input energy is imposed on all inputs, a comparison among the inputs using a criterion which is a function of D is inherently unfair because a wide range of maximum allowable input amplitudes and maneuver duration can give the same input energy. The inputs compared in this work have the same maneuver duration and maximum allowable input amplitudes, insofar as possible.

Similarly, the dispersion matrix $\mathbf{D}$ depends nonlinearly on the states, which are often the same as the outputs. Therefore, output amplitudes must be comparable if an input design comparison is to be focused only on the merits of the input forms. For this reason, as well as to ensure validity of the assumed model structure, the inputs were designed to produce comparable output amplitudes. If the maneuver duration, input amplitudes, and output amplitudes are not the same for all input designs being compared, it is possible to arrange matters so that almost any chosen input form will appear to be the best, based on a criterion function that depends on $\mathbf{D}$.

For the optimal input design, the flight test maneuver duration $T=N \Delta t$ was fixed $a$ priori due to practical time constraints of the flight test and an analysis of the rate of decrease of the Cramér-Rao bounds with increasing maneuver time using the optimized input. The cost function to be minimized was the sum of squares of the Cramér-Rao bounds for the parameter standard errors,

$$
J=\sum_{j=1}^{n_{p}} \sigma_{j}^{2}=\operatorname{Tr}\left[\mathbf{M}^{-1}\right]=\operatorname{Tr}[\mathbf{D}] \quad \text { for a given } T
$$

Another formulation of the cost can be defined to design the input for minimum flight test time to achieve specific goals for the Cramér-Rao bounds ${ }^{14}$.

The optimal input applied to the dynamic system described by Eqs. (1)-(5) minimizes the cost function in Eq. (14), subject to the constraints in Eqs. (6) and (7).

\section{Optimal Input Solution Methodology}

The optimization problem posed in the last section is difficult to solve in general. At this point, considerations particular to optimal input design for aircraft parameter estimation problems were invoked in order to limit the allowable input form to square waves only. Among these considerations was analytical work on a similar problem ${ }^{8}$, which indicated that the optimal input should be "bang-bang" (i.e., a full amplitude switching input). In addition, simple implementation by either computer or the pilot was desired. Finally, previous flight test evaluations ${ }^{11,12}$ demonstrated that inputs similar to square waves were superior to sinusoidal inputs for parameter estimation experiments, largely due to their richer frequency spectra.

For the above reasons, and to make the optimization problem tractable, input forms were limited to square waves only; i.e., only full positive, full negative, or zero amplitude was allowed for any input at any time. Full input amplitude was used in order to excite the system as much as possible. Choice of the pulse timing and having zero amplitude available gave the optimizer the ability to use full input amplitudes without exceeding output amplitude constraints. With the above restriction on the input form, the problem becomes a high order combinatorial problem involving output amplitude constraints, which is well-suited to solution by the method of dynamic programming.

Dynamic programming is essentially a very efficient method for doing a global exhaustive search. Arbitrary dynamics such as control surface actuator dynamics, feedback control, and general nonlinear models can therefore be included inside the optimization without difficulty. The result obtained is a globally optimal square wave input obtained in a single pass solution. In addition, the optimal input design technique includes provisions to adjust the input possibilities at certain times in order to account for practical limitations on frequency content of the input, such as avoiding structural resonance frequencies. The dynamic programming solution smoothly handles the multiple input problem, since this just changes the number of square wave input possibilities. Keeping the system responses within the range of output space for which the assumed linear model is valid can be handled directly with dynamic programming by discarding any input sequence whose output trajectory exceeds the constraint limits. More details on the dynamic programming solution method can be found in Refs. [14] and [15].

\section{Aircraft and Test Procedure}

The F-18 High Alpha Research Vehicle (HARV) is a modified F/A-18 fighter ${ }^{19}$. The flight test inputs were implemented by a computer-controlled On-Board Excitation System (OBES).

The pilot initiated each run by selecting a pre-programmed maneuver using buttons on a Digital Display Interface (DDI) inside the cockpit. The aircraft was then brought to the desired trimmed flight condition and an engage/disengage button on the DDI was pressed to initiate the maneuver. Perturbation inputs were added directly to the appropriate control surface actuator command by the OBES, with the feedback control system still operating. The pilot held stick and rudder deflections constant at the trimmed values until the maneuver was 
complete. The maneuver could be disengaged manually by the pilot toggling the engage/disengage button, or automatically by the research flight control system, based on g-limits, etc. The automatic flight control implemented for the flight test maneuvers studied here was the NASA-1 control law in Thrust Vectoring (TV) mode 20. Pre-programmed commands to the control surface actuators were standard 3-2-1-1 inputs, doublets, or square wave inputs obtained from the optimal input design technique described above.

Various downlink data transmission rates were employed on the F-18 HARV aircraft, but all of the data used for analysis was converted to a common sampling rate of $40 \mathrm{~Hz}$. Corrections were applied to the angle of attack, sideslip angle, and linear accelerometer measurements to account for sensor offsets from the center of gravity, and the angle of attack measurement was corrected for upwash. Data compatibility analysis ${ }^{21}$ revealed the need for a scale factor correction on the angle of attack and sideslip angle measurements from the wing tip vane, and small bias error corrections on the measurements from the rate gyros and accelerometers.

\section{$\underline{\text { Results }}$}

A priori linear models used for the input design were derived from a nonlinear batch simulation of the F-18 $\mathrm{HARV}^{22}$, which uses a wind tunnel database for the aerodynamics. Noise variance estimates for the input design were obtained from previous flight test data records using an optimal Fourier smoothing technique ${ }^{23}$. The models used for parameter estimation from flight test data were identical in structure to the a priori models, except that the a priori models did not include linear accelerometer outputs.

For lateral-directional aircraft dynamics, the state vector $\mathbf{x}$, input vector $\mathbf{u}$, and output vector $\mathbf{y}$ in Eqs. (1)-(4) are defined by

$$
\begin{gathered}
\mathbf{x}=\left[\begin{array}{cccc}
\beta & p & r & \phi
\end{array}\right]^{T} \quad \mathbf{u}=\left[\begin{array}{lll}
\delta_{r} & \delta_{a} & 1
\end{array}\right]^{T} \\
\mathbf{y}=\left[\begin{array}{lllll}
\beta & p & r & \phi & a_{y}
\end{array}\right]^{T}
\end{gathered}
$$

System matrices $\mathbf{A}, \mathbf{B}, \mathbf{C}$, and $\mathbf{D}$ contain the model parameters:

$$
\mathbf{A}=\left[\begin{array}{cccc}
\mathrm{Y}_{\beta} & \sin \alpha_{o} & -\cos \alpha_{o} & \left(g / V_{\mathrm{o}}\right) \cos \Theta_{\mathrm{o}} \\
\mathrm{L}_{\beta} & \mathrm{L}_{p} & \mathrm{~L}_{r} & 0 \\
\mathrm{~N}_{\beta} & \mathrm{N}_{p} & \mathrm{~N}_{r} & 0 \\
0 & 1 & \tan \Theta_{\mathrm{o}} & 0
\end{array}\right]
$$

$$
\begin{gathered}
\mathbf{B}=\left[\begin{array}{ccc}
\mathrm{Y}_{\delta_{r}} & \mathrm{Y}_{\delta_{a}} & \mathrm{Y}_{o} \\
\mathrm{~L}_{\delta_{r}} & \mathrm{~L}_{\delta_{a}} & \mathrm{~L}_{o} \\
\mathrm{~N}_{\delta_{r}} & \mathrm{~N}_{\delta_{a}} & \mathrm{~N}_{o} \\
0 & 0 & 0
\end{array}\right] \\
\mathbf{C}=\left[\begin{array}{cccc}
1 & 0 & 0 & 0 \\
0 & 1 & 0 & 0 \\
0 & 0 & 1 & 0 \\
0 & 0 & 0 & 1 \\
Y_{\beta} V_{\mathrm{o}} / g & 0 & 0 & 0
\end{array}\right] \\
\mathbf{D}=\left[\begin{array}{cccc}
0 & & & \\
0 & 0 & 0 \\
0 & 0 & 0 \\
0 & 0 & 0 \\
Y_{\delta_{r}} V_{\mathrm{o}} / g & Y_{\delta_{a}} V_{\mathrm{o}} / g & a_{z_{o}}
\end{array}\right]
\end{gathered}
$$

For longitudinal aircraft short period dynamics, the state vector $\mathbf{x}$, input vector $\mathbf{u}$, and output vector $\mathbf{y}$ in Eqs. (1)-(4) are defined by

$$
\mathbf{x}=\left[\begin{array}{ll}
\alpha & q
\end{array}\right]^{T} \quad \mathbf{u}=\left[\begin{array}{ll}
\delta_{s} & 1
\end{array}\right]^{T} \quad \mathbf{y}=\left[\begin{array}{lll}
\alpha & q & a_{z}
\end{array}\right]^{T}(21)
$$

System matrices containing the model parameters are:

$$
\begin{gathered}
\mathbf{A}=\left[\begin{array}{cc}
\mathrm{Z}_{\alpha} & 1 \\
\mathrm{M}_{\alpha} & \mathrm{M}_{q}
\end{array}\right] \\
\mathbf{B}=\left[\begin{array}{ll}
\mathrm{Z}_{\delta_{s}} & \mathrm{Z}_{o} \\
\mathrm{M}_{\delta_{s}} & \mathrm{M}_{o}
\end{array}\right] \\
\mathbf{C}=\left[\begin{array}{cc}
1 & 0 \\
0 & 1 \\
\mathrm{Z}_{\alpha} V_{\mathrm{o}} / g & 0
\end{array}\right] \\
\mathbf{D}=\left[\begin{array}{cc}
0 & 0 \\
0 & 0 \\
\mathrm{Z}_{\delta_{s}} V_{\mathrm{o}} / g & a_{z_{o}}
\end{array}\right]
\end{gathered}
$$

The data analysis was done using output error maximum likelihood parameter estimation in the time domain ${ }^{2,3}$. The Cramér-Rao bounds for the parameter standard errors were computed from the square root of the diagonal elements of the dispersion matrix $\mathbf{D}$ in Eq. (9). Ordinarily, these values should be corrected for colored residuals 3 . The correction was not done here because the particular frequency content of the output residuals 
(usually from model structure errors) introduces a confounding factor in the comparison of data information content from different input forms. In the parameter estimation context, the estimated noise covariance matrix $\mathbf{R}$ used in Eq. (8) provides a measure of the mean square model fit error for all the outputs, and this was considered appropriate for the present analysis. The model structure was the same for the compared maneuvers, so that the number of parameters estimated from each data record was identical. All data analysis and parameter estimates used radians for angular measure, but the plots were made using degrees.

The first input design was a lateral-directional case using the OBES to implement sequential rudder and aileron inputs. The flight condition was 5 degrees angle of attack and altitude of approximately 25,000 feet. The model was given by Eqs. (1)-(5) and (15)-(20). Perturbation input and output amplitude constraints were:

$$
\begin{array}{ll}
\left|\delta_{r}\right| \leq 4.0 \mathrm{deg} & \left|\delta_{a}\right| \leq 2.5 \mathrm{deg} \\
|\beta| \leq 5.0 \mathrm{deg} & |\phi| \leq 32.0 \mathrm{deg}
\end{array}
$$

To effect a fair comparison, the 3-2-1-1 and optimal inputs were designed with the same input amplitude constraints in (26), using the same a priori model, and satisfying the same output amplitude constraints in (26).

The 3-2-1-1 inputs were designed by matching the frequency of the "2" pulse to the frequency of the dominant oscillatory mode for the a priori model, and adjusting amplitudes and control sequence timing so that the chosen output amplitude constraints were satisfied. Optimal inputs were designed with a computer program that implemented the optimal input design procedure described above ${ }^{14}$. The duration of each maneuver was 24 seconds.

The solid lines in Figures 1 and 2 show the input and output time histories measured in flight for the OBES lateral-directional 3-2-1-1 and optimal inputs at 5 degrees angle of attack. The desired input forms were distorted somewhat by the feedback control system, as can be seen in the figures. The distortion of the input forms by the lateral-directional feedback control system was not accounted for in the design process for either input. Figures 1 and 2 show that the maximum input and output amplitudes for these two maneuvers were very nearly the same, and the length of each maneuver was the same. The maneuvers were run in immediate succession on the same flight. With the model structure held fixed for the data analysis on each maneuver, any differences in the resulting model parameter accuracies can be attributed to effect of the input form.

Parameter estimation results for the OBES lateral-directional 3-2-1-1 and optimal inputs at 5 degrees angle of attack are given in Table 1 . Column 1 in Table 1 lists the model parameters, column 2 contains the parameter estimates from the 3-2-1-1 input, and column 3 contains the estimated Cramér-Rao lower bounds for the parameter standard errors using the 3-2-1-1 input. Columns 4 and 5 contain the corresponding results for the optimal square wave input. The values in parentheses in column 5 are the percent change in the Cramér-Rao bound for the parameter standard error, based on the 3-2-1-1 value. The optimal input reduced parameter standard errors (equivalently, increased parameter accuracy) by an average $20 \%$, with lower parameter standard errors for every estimated parameter. Parameter estimates in columns 2 and 4 of Table 1 are generally in good agreement.

The dashed lines in Figures 1 and 2 are the model responses computed using the measured inputs and the estimated model parameters from columns 2 and 4 of Table 1. The match is very good in both cases. The percent error of the a priori parameter values relative to the parameter values estimated from flight test data (computed as the average of values in columns 2 and 4 of Table 1) varied from $4.2 \%$ to $65.1 \%$, with an average value of $24.2 \%$. Nevertheless, both input design methods based on the a priori model produced experimental data with excellent information content, as evidenced by the low standard error bounds in Table 1.

Symmetric stabilator input designs implemented by OBES for longitudinal model identification are shown in Figures 3 and 4. In this case, the distortion of the input forms by the feedback control was accounted for in the a priori model by including a linear model of the feedback control identified from the nonlinear simulation. The flight condition was again 5 degrees angle of attack and altitude of approximately 25,000 feet. The model is given by Eqs. (1)-(5) and (21)-(25). The same methods were used for the input designs and the data analysis, except that the optimal input design was allowed a higher input amplitude than the 3-2-1-1 input. This was done to investigate the capability available with the optimal input design routine to use higher input amplitudes for increased parameter accuracies while maintaining the same output amplitude constraints. This flexibility is not available with the 3-2-1-1 input because of its fixed form. Perturbation input and output amplitude constraints were:

$$
\begin{aligned}
& |\alpha| \leq 3.0 \mathrm{deg} \\
& \left|\delta_{s}\right| \leq 3.0 \mathrm{deg} \quad \text { for the } 3-2-1-1 \text { input } \\
& \left|\delta_{s}\right| \leq 4.0 \mathrm{deg} \quad \text { for the optimal input }
\end{aligned}
$$

Each maneuver lasted 26 seconds, and the maneuvers were run in immediate succession on the same flight. The left sides of Figures 3 and 4 show the significant distortion of the stabilator commands resulting from the longitudinal feedback control. Parameter estimation 
results are given in Table 2 using the same format as for Table 1. The parameter accuracies are now improved by an average $72 \%$ using the optimal input compared to the 3-2-1-1 input, while the output amplitude remains very nearly within the constraint. The right sides of Figures 3 and 4 indicate a good match between the measured outputs and the model responses using the measured inputs and the estimated model parameters from columns 2 and 4 of Table 2. The estimates of pitching moment parameters in columns 2 and 4 of Table 2 do not agree. Lower parameter standard error bounds for the optimal input indicate that the pitching moment parameter estimates from the optimal input should be more accurate. To check this, a different maneuver at the same flight condition was used to investigate the prediction capability of the models using the parameters in Table 2. Figure 5 shows measured and predicted pitch rate response using the model parameters from Table 2 with the same model structure used before. The stabilator input (not shown) was a perturbation input with amplitude approximately \pm 5 degrees from the trim value of 2 degrees. The stabilator input was applied to both models to produce the prediction responses plotted with the measured response. The prediction using the parameters estimated from the 3-2-1-1 input (shown on the left side of Figure 5) was less accurate than the prediction using the parameters from the optimal input (shown on the right side of Figure 5), both in frequency and amplitude. This result gives confidence that the parameters estimated from the optimal input maneuver are indeed more accurate, as indicated by the computed Cramér-Rao bounds.

Finally, two longitudinal maneuvers flown at 20 degrees angle of attack and approximately 25,000 feet altitude were studied to compare the optimal input design method to a sequence of doublets. Each maneuver lasted 16 seconds. The left side of Figure 6 shows the control surface deflections for the doublet sequence. Individual doublets were commanded for the trailing edge flap, symmetric aileron, and stabilator, in sequence. The traces in Figure 6 are the measured control surface deflections, which include the effect of the feedback control system. The optimal input design used only the stabilator. The left side of Figure 7 shows measured stabilator deflection for the optimal square wave input, which was also distorted by the feedback control. The two maneuvers were run on different flights on different days, so the data analysis was done using non-dimensional stability and control derivatives. The model structure was the same as in the last example, except that the $C_{Z_{q}}$ parameter was included for this higher angle of attack flight condition, and extra control derivatives were included for the doublet sequence maneuver, since three controls were used. The additional controls put the doublet sequence maneuver at a disadvantage in the comparison because of the additional parameters to be estimated from the data record. This disadvantage was offset somewhat by the wider variety of excitation capability available using different control effectors. The stabilator amplitude perturbations from trim were approximately the same for the two maneuvers, as can be seen from Figures 6 and 7. An imprecise initial trim caused the nominal angle of attack to drift throughout the optimal input maneuver in Figure 7. The right sides of Figures 6 and 7 show that the maneuvers produced roughly the same angle of attack perturbations and that the model matched the measurements very well for both maneuvers. Table 3 gives the results of the data analysis in a format similar to the other tables. The results indicate that the optimal input maneuver lowered the estimated parameter standard errors by an average $64 \%$ compared to the doublet sequence maneuver.

\section{Concluding Remarks}

The expense associated with flight testing modern aircraft dictates that flight test data for modeling purposes be collected as efficiently as possible. In this work, the impact of input form on estimated parameter accuracy was investigated for three leading input design techniques: 3-2-1-1, doublets, and the optimal square wave. The tests were conducted on the F-18 HARV at 5 and 20 degrees angle of attack. Comparisons were done on an equal basis, and it was found that the optimal input decreased estimated parameter standard errors (equivalently, increased estimated parameter accuracy) by an average $20 \%$ compared to the 3-2-1-1 input. The decrease in estimated parameter standard errors improved to an average $72 \%$ using higher input amplitudes in the optimal input design while maintaining flight condition. Compared to a doublet sequence, the optimal input decreased estimated parameter standard errors by an average 64\%. For all the comparisons, every individual parameter was estimated more accurately using the optimal square wave input.

The results of this investigation indicate that a properly designed 3-2-1-1 input can give good performance relative to the optimal square wave. Optimal square wave input designs demonstrated increased data information content in all cases studied, but the optimal input design technique is perhaps most valuable because of its ability to address practical design issues. Examples include an automated ability to limit output amplitude excursions during the flight test maneuver, good robustness to errors in the a priori model and to distortions in the realized input form, and the design flexibility to investigate the impact of changes in the conditions or constraints of the input design, such as available maneuver time, control surface rate limits, or input/output amplitude constraints. Such changes can be evaluated in terms of estimated parameter accuracies, using the single pass global optimizer imbedded in the optimal input design procedure. Some of these capabilities were demonstrated in this work using flight test results. 


\section{Acknowledgments}

Discussions with Dr. Vladislav Klein of the George Washington University contributed to the work presented here. Useful technical reviews were provided by Don Riley and Dr. Pat Murphy of NASA Langley Research Center. Flight tests were carried out at NASA Dryden Flight Research Center.

\section{References}

1. Williams, J.N., Ham, J.A., and Tischler, M.B. "Flight Test Manual, Rotorcraft Frequency Domain Flight Testing", AQTD Project No. 93-14, U.S. Army Aviation Technical Test Center, Edwards AFB, California. September 1995.

2. Maine, R.E. and Iliff, K.W., "Application of Parameter Estimation to Aircraft Stability and Control - The Output-Error Approach", NASA RP 1168, June 1986.

3. Morelli, E.A. and Klein, V., "Determining the Accuracy of Maximum Likelihood Parameter Estimates with Colored Residuals", NASA CR 194893, March 1994.

4. Stepner, D.E. and Mehra, R.K., "Maximum Likelihood Identification and Optimal Input Design for Identifying Aircraft Stability and Control Derivatives", NASA CR-2200, March 1973.

5. Mehra, R. K., "Optimal Input Signals for Parameter Estimation in Dynamic Systems - Survey and New Results", IEEE Transactions on Automatic Control, Vol. AC-19, No. 6, December 1974.

6. Gupta, N.K. and Hall, W.E., Jr., "Input Design for Identification of Aircraft Stability and Control Derivatives", NASA CR-2493, February, 1975.

7. Mehra, R. K. and Gupta, N. K., "Status of Input Design for Aircraft Parameter Identification", AGARD-CP-172, paper 12, 1975.

8. Chen, R. T. N., "Input Design for Aircraft Parameter Identification: Using Time-Optimal Control Formulation", AGARD-CP-172, paper 13, 1975.

9. Gupta, N.K. and Hall, W.E., Jr., "Model Structure Determination and Test Input Selection for Identification of Nonlinear Regimes", Report ONRCR215-213-5, Office of Naval Research, Arlington, VA, February, 1976.

10. Gupta, N.K., Mehra, R.K. and Hall, W.E., Jr., "Application of Optimal Input Synthesis to Aircraft Parameter Identification", Journal of Dynamic Systems, Measurement and Control, Vol. 98, No. 2, pp. 139-45, June 1976.
11. Plaetschke, E. and Schulz, G., "Practical Input Signal Design", AGARD-LS-104, paper 3, 1979.

12. Plaetschke, E., Mulder, J.A., and Breeman, J.H., "Flight Test Results of Five Input Signals for Aircraft Parameter Identification", Proc. Sixth IFAC Symposium on Identification and System Parameter Estimation, Washington, D.C., January 7-11, 1982, Vol. 2, pp. 1149-1154, Pergamon Press, Oxford and New York, 1983.

13. Mulder, J. A., "Design and Evaluation of Dynamic Flight Test Manoeuvres", Report LR-497, Delft University of Technology, Department of Aerospace Engineering, Delft, The Netherlands, 1986.

14. Morelli, E. A., "Practical Input Optimization for Aircraft Parameter Estimation Experiments", Sc.D. dissertation, George Washington University JIAFS, July 1990; also published as NASA CR-191242, May 1993.

15. Morelli, E. A. and Klein, V., "Optimal Input Design for Aircraft Parameter Estimation Using Dynamic Programming Principles", AIAA Paper 90-2801, AIAA Atmospheric Flight Mechanics Conference, Portland, OR. August 1990.

16. Morelli, E.A., "Flight Test Validation of Optimal Input Design using Pilot Implementation", IFAC paper IFAC-559, 10th IFAC Symposium on System Identification, Copenhagen, Denmark, July 1994.

17. Morelli, E.A.: Optimal Input Design for Closed Loop Modeling at High Angles of Attack. AIAA paper 96-3418, AIAA Atmospheric Flight Mechanics Conference. San Diego, California. July 1996.

18. van der Linden, C.A.A.M., Mulder, J.A., and Sridhar, J.K. "Recent Developments in Aircraft Parameter Identification at Delft University of Technology - Optimal Input Design", in Cook, M.V., and Rycroft, M.J. (Eds.), Aerospace Vehicle Dynamics and Control, Clarendon Press, Oxford, 1994, pp. 259-84.

19. Kempel, R. "F-18 High Alpha Research Vehicle Description", Internal Document, NASA Dryden Flight Research Facility, Edwards, California.

20. HARV Control Law Design Team. "Design Specification for a Thrust-Vectoring, ActuatedNose-Strake Flight Control Law for the High-Alpha Research Vehicle", NASA TM 110217, November 1995.

21. Klein, V., and Morgan, D.R. "Estimation of Bias Errors in Measured Airplane Responses using Maximum Likelihood Method", NASA TM-89059, January 1987. 
22. Messina, M.D., et al., "Simulation Model of the F/A-18 High Angle-of-Attack Research Vehicle Utilized for the Design of Advanced Control Laws", NASA TM-110216, May 1996.

23. Morelli, E.A. : Estimating Noise Characteristics from Flight Test Data using Optimal Fourier Smoothing. Journal of Aircraft, Vol. 32, No. 4, July-August 1995, pp. 689-695.

Table 1 Maximum Likelihood Results for Lateral-Directional OBES Maneuvers, $\alpha=5^{\circ}$

$$
3-2-1-1
$$

optimal

\begin{tabular}{|c|c|c|c|c|}
\hline $\begin{array}{c}\text { Parameter } \\
\text { (a priori) }\end{array}$ & Estimate & $\begin{array}{c}\text { Error } \\
\text { Bound }\end{array}$ & Estimate & $\begin{array}{c}\text { Error } \\
\text { Bound } \\
(\% \text { change })\end{array}$ \\
\hline $\begin{array}{c}\mathrm{Y}_{\beta} \\
(-0.1316)\end{array}$ & -0.0970 & 0.00130 & -0.0859 & $\begin{array}{c}0.00121 \\
(-7.2)\end{array}$ \\
\hline $\begin{array}{c}\mathrm{Y}_{\delta_{r}} \\
(0.0285)\end{array}$ & 0.0304 & 0.00092 & 0.0327 & $\begin{array}{c}0.00079 \\
(-14.1)\end{array}$ \\
\hline $\begin{array}{c}\mathrm{Y}_{\delta_{a}} \\
(0.0053) \\
\end{array}$ & 0 & $\dagger$ & 0 & $\dagger$ \\
\hline $\begin{array}{c}\mathrm{L}_{\beta} \\
(-11.56) \\
\end{array}$ & -11.376 & 0.04807 & -10.764 & $\begin{array}{c}0.03709 \\
(-22.8) \\
\end{array}$ \\
\hline $\begin{array}{c}\mathrm{L}_{p} \\
(-1.592)\end{array}$ & -1.8120 & 0.00695 & -1.7998 & $\begin{array}{c}0.00549 \\
(-21.1)\end{array}$ \\
\hline $\begin{array}{c}\mathrm{L}_{r} \\
(0.5462)\end{array}$ & 0.3396 & 0.02238 & 0.1727 & $\begin{array}{c}0.02005 \\
(-10.4)\end{array}$ \\
\hline $\begin{array}{c}\mathrm{L}_{\delta_{r}} \\
(1.910)\end{array}$ & 2.3074 & 0.03979 & 1.8768 & $\begin{array}{c}0.03155 \\
(-20.7)\end{array}$ \\
\hline $\begin{array}{c}\mathrm{L}_{\delta_{a}} \\
(-15.81) \\
\end{array}$ & -19.480 & 0.06234 & -17.470 & $\begin{array}{c}0.04410 \\
(-29.3) \\
\end{array}$ \\
\hline $\begin{array}{c}\mathrm{N}_{\beta} \\
(2.139) \\
\end{array}$ & 1.2807 & 0.00394 & 1.3120 & $\begin{array}{c}0.00284 \\
(-27.9)\end{array}$ \\
\hline $\begin{array}{c}\mathrm{N}_{p} \\
(-0.0085)\end{array}$ & 0 & $\dagger$ & 0 & $\dagger$ \\
\hline $\begin{array}{c}\mathrm{N}_{r} \\
(-0.0940)\end{array}$ & -0.1027 & 0.00214 & -0.0436 & $\begin{array}{c}0.00189 \\
(-11.7) \\
\end{array}$ \\
\hline $\begin{array}{c}\mathrm{N}_{\delta_{r}} \\
(-1.223)\end{array}$ & -1.3924 & 0.00558 & -1.3450 & $\begin{array}{c}0.00427 \\
(-23.5)\end{array}$ \\
\hline $\begin{array}{c}\mathrm{N}_{\delta_{a}} \\
(0.2444)\end{array}$ & 0.1738 & 0.00378 & 0.2383 & $\begin{array}{c}0.00278 \\
(-26.6)\end{array}$ \\
\hline
\end{tabular}

$\dagger=$ parameter dropped in model structure determination
Table 2 Maximum Likelihood Results for Longitudinal OBES Maneuvers, $\alpha=5^{\circ}$

$$
3-2-1-1
$$

\begin{tabular}{|c|c|c|c|c|}
\hline $\begin{array}{c}\text { Parameter } \\
\text { ( a priori) }\end{array}$ & Estimate & $\begin{array}{l}\text { Error } \\
\text { Bound }\end{array}$ & Estimate & $\begin{array}{c}\text { Error } \\
\text { Bound } \\
\text { (\% change })\end{array}$ \\
\hline $\begin{array}{c}\mathrm{Z}_{\alpha} \\
(-0.5832)\end{array}$ & -0.5940 & 0.0126 & -0.6050 & $\begin{array}{l}0.0047 \\
(-62.8) \\
\end{array}$ \\
\hline $\begin{array}{c}\mathrm{Z}_{\delta_{s}} \\
(-0.1093)\end{array}$ & -0.0378 & 0.0063 & -0.0789 & $\begin{array}{l}0.0032 \\
(-49.3)\end{array}$ \\
\hline $\begin{array}{c}\mathrm{M}_{\alpha} \\
(-2.2600)\end{array}$ & -4.543 & 0.080 & -2.195 & $\begin{array}{c}0.012 \\
(-85.1) \\
\end{array}$ \\
\hline $\begin{array}{c}\mathrm{M}_{q} \\
(-0.2927)\end{array}$ & -4.746 & 0.109 & -1.341 & $\begin{array}{c}0.014 \\
(-86.8)\end{array}$ \\
\hline $\begin{array}{c}\mathrm{M}_{\delta_{s}} \\
(-6.0380)\end{array}$ & -5.482 & 0.104 & $\begin{array}{l}-4.597 \\
\end{array}$ & $\begin{array}{c}0.024 \\
(-76.4)\end{array}$ \\
\hline
\end{tabular}

optimal

Table 3 Maximum Likelihood Results

\begin{tabular}{|c|c|c|c|c|}
\hline & \multicolumn{2}{|c|}{ Doublets } & \multicolumn{2}{|c|}{ Optimal } \\
\hline Parameter & Estimate & $\begin{array}{c}\text { Error } \\
\text { Bound }\end{array}$ & Estimate & $\begin{array}{c}\text { Error } \\
\text { Bound } \\
(\% \text { change }) \\
\end{array}$ \\
\hline$\overline{\overline{C_{Z_{\alpha}}}}$ & -3.104 & 0.143 & -2.087 & $\begin{array}{c}0.066 \\
(-53.5) \\
\end{array}$ \\
\hline$C_{Z_{q}}$ & -45.17 & 6.91 & -71.58 & $\begin{array}{c}3.72 \\
(-46.1)\end{array}$ \\
\hline$C_{Z_{\delta_{f}}}$ & -0.4422 & 0.0165 & $*$ & \\
\hline$C_{Z_{\delta_{a_{s y m}}}}$ & -0.2243 & 0.0138 & $*$ & \\
\hline$C_{Z_{\delta_{s}}}$ & -0.7783 & 0.1002 & -0.8846 & $\begin{array}{l}0.0400 \\
(-60.0) \\
\end{array}$ \\
\hline$C_{M_{\alpha}}$ & -0.1792 & 0.0179 & -0.3313 & $\begin{array}{l}0.0037 \\
(-79.3)\end{array}$ \\
\hline$\overline{C_{M_{q}}}$ & -54.08 & 1.14 & -16.10 & $\begin{array}{c}0.31 \\
(-72.4) \\
\end{array}$ \\
\hline$C_{M_{\delta_{f}}}$ & $\dagger$ & & $*$ & \\
\hline$C_{M_{\delta_{a_{s y m}}}}$ & -0.0953 & 0.0019 & $*$ & \\
\hline$C_{M_{\delta_{s}}}$ & -1.159 & 0.019 & -0.646 & $\begin{array}{c}0.005 \\
(-71.4)\end{array}$ \\
\hline
\end{tabular}
for Longitudinal OBES Maneuvers, $\alpha=20^{\circ}$ 

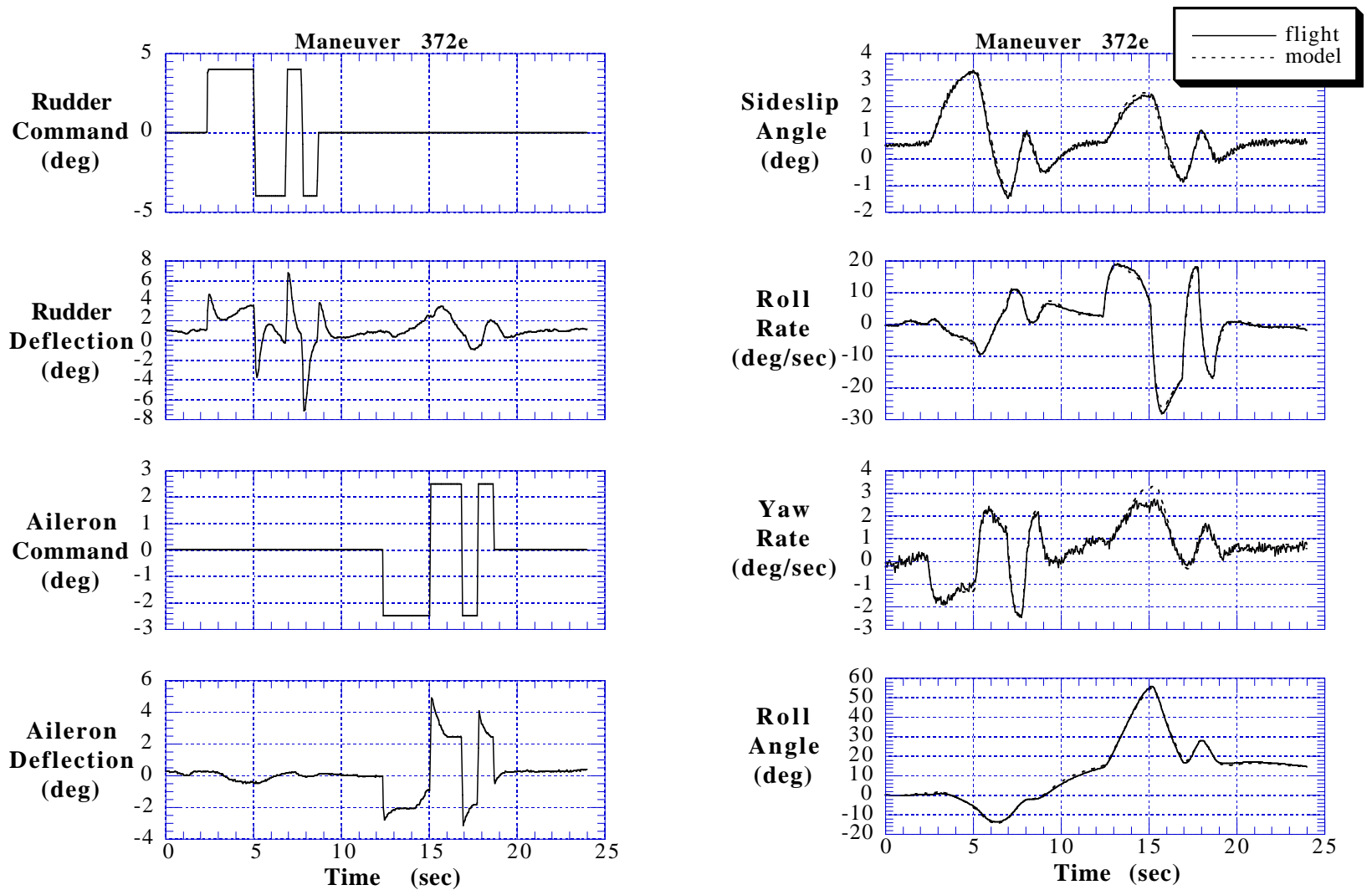

Figure 1 Lateral-Directional 3-2-1-1 Input, $\alpha=5^{\circ}$
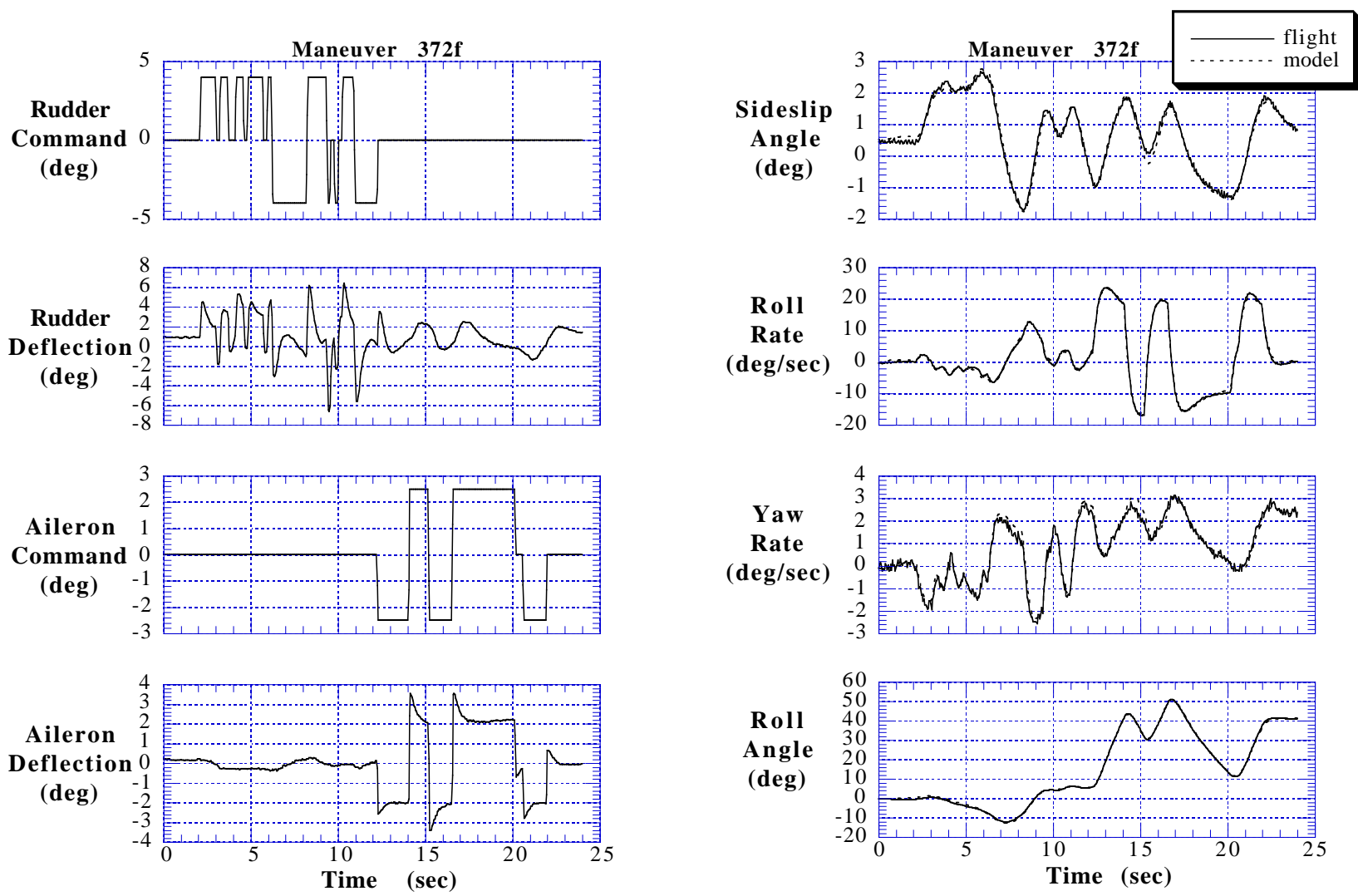

$\underline{\text { Figure } 2}$ Lateral-Directional Optimal Input, $\alpha=5^{\circ}$ 

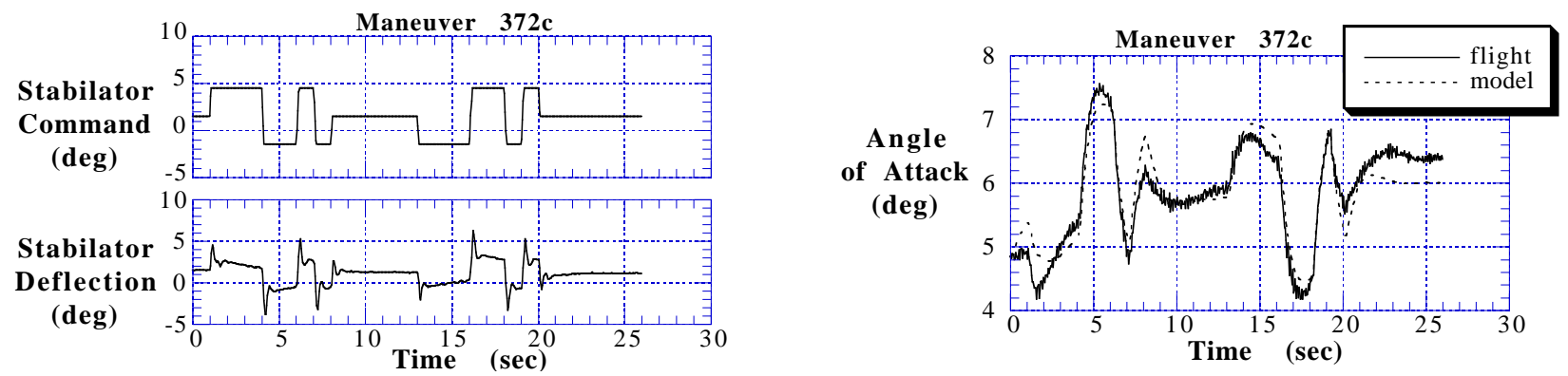

Figure 3 Longitudinal 3-2-1-1 Input, $\alpha=5^{\circ}$
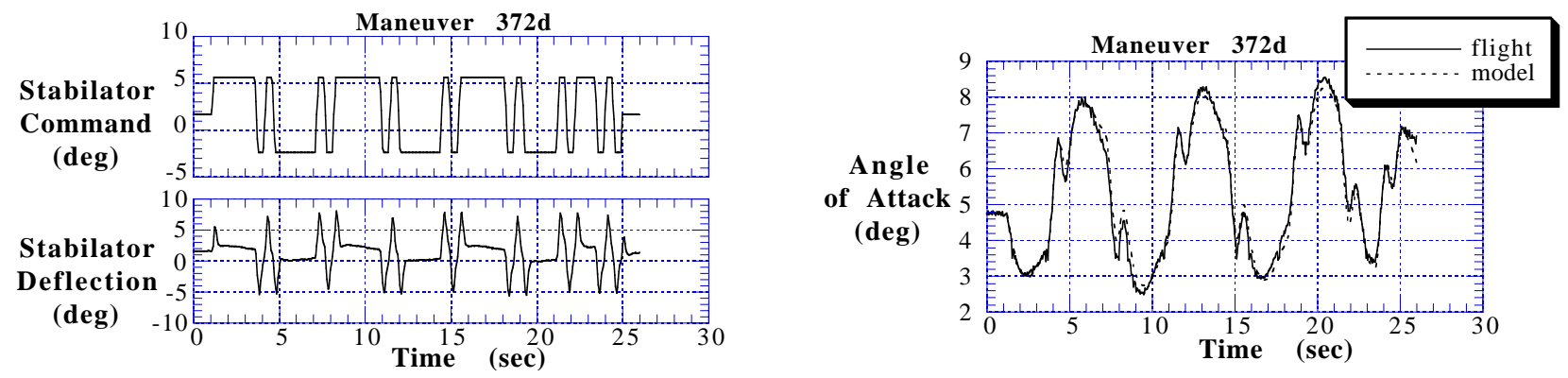

Figure 4 Longitudinal Optimal Input, $\alpha=5^{\circ}$
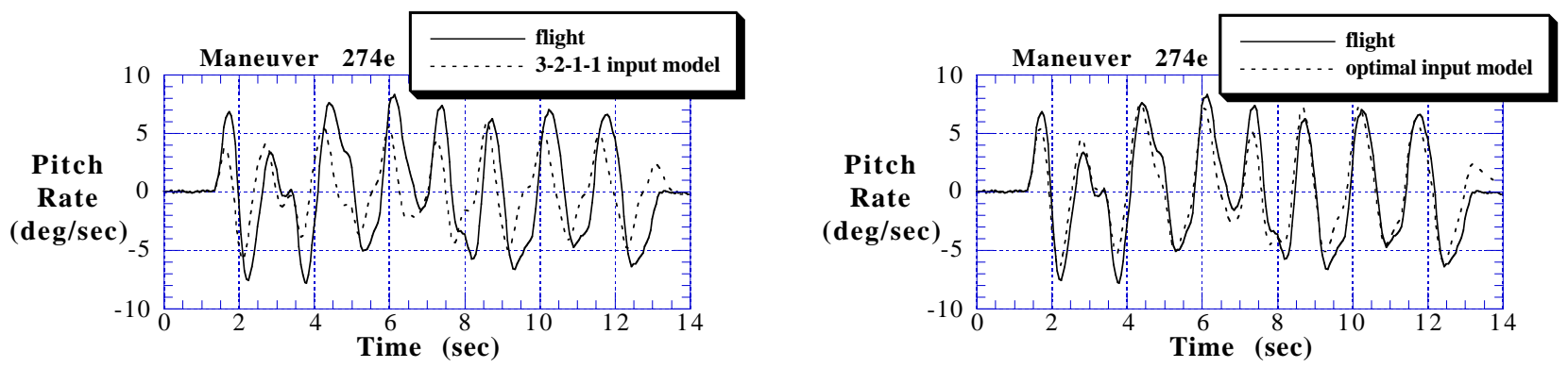

Figure 5 Longitudinal Prediction Case, $\alpha=5^{\circ}$
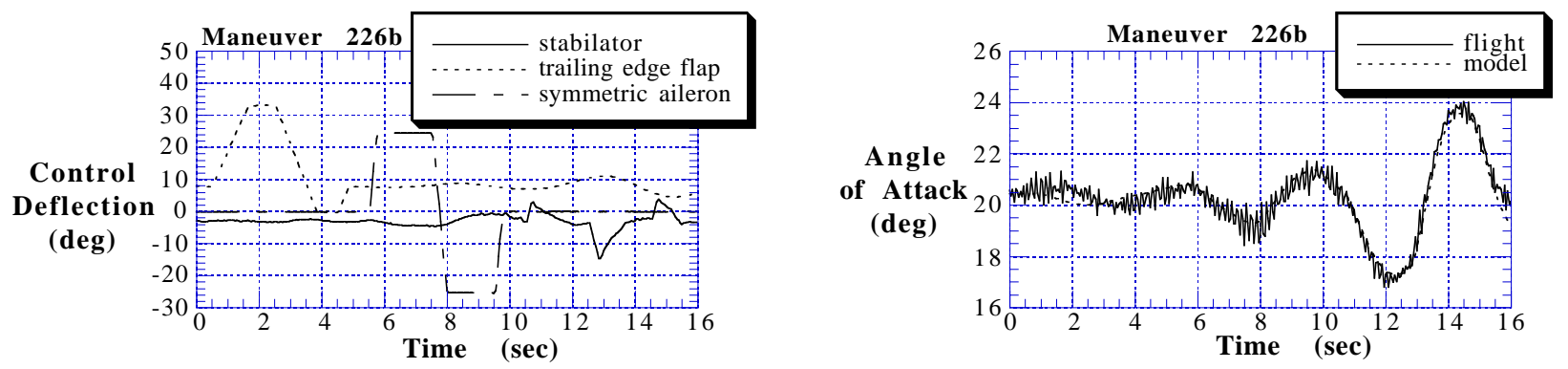

Figure 6 Longitudinal Doublet Input, $\alpha=20^{\circ}$
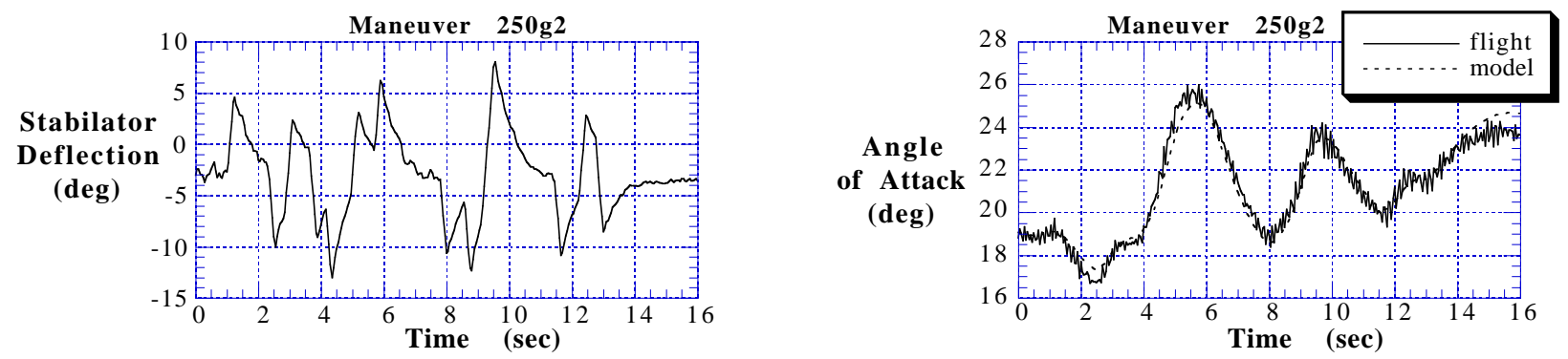
$\underline{\text { Figure } 7}$ Longitudinal Optimal Input, $\alpha=20^{\circ}$
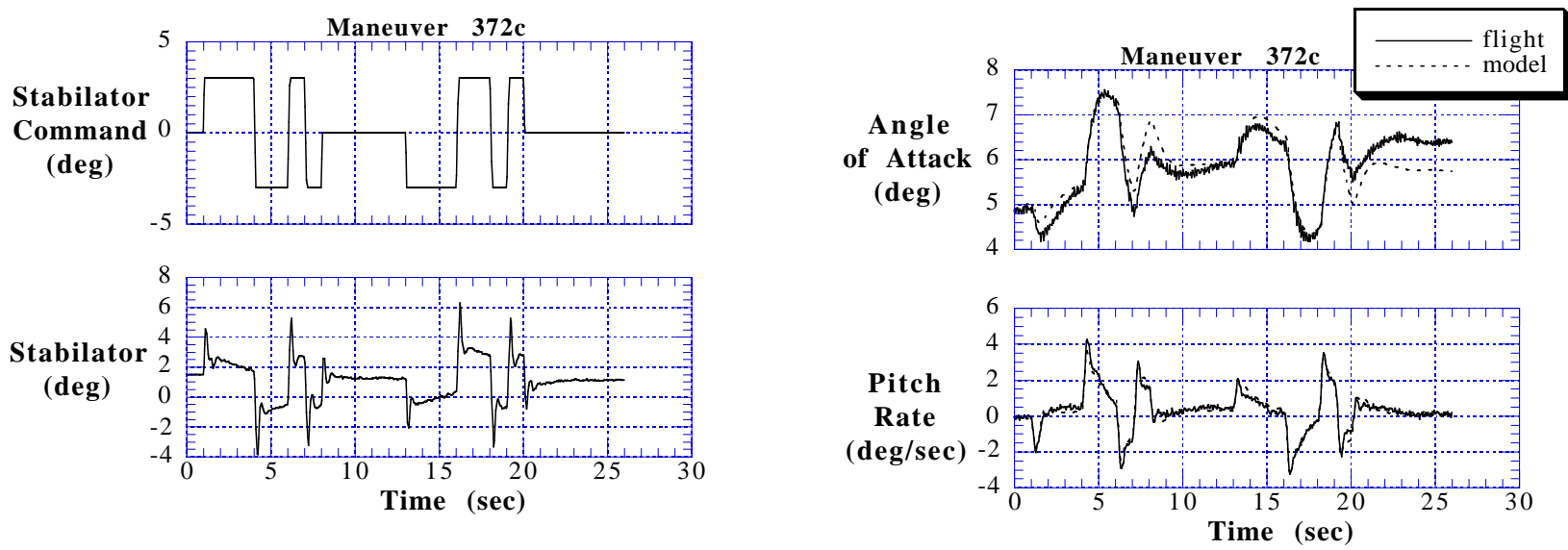

Figure 3 Longitudinal 3-2-1-1 Input, $\alpha=5^{\circ}$
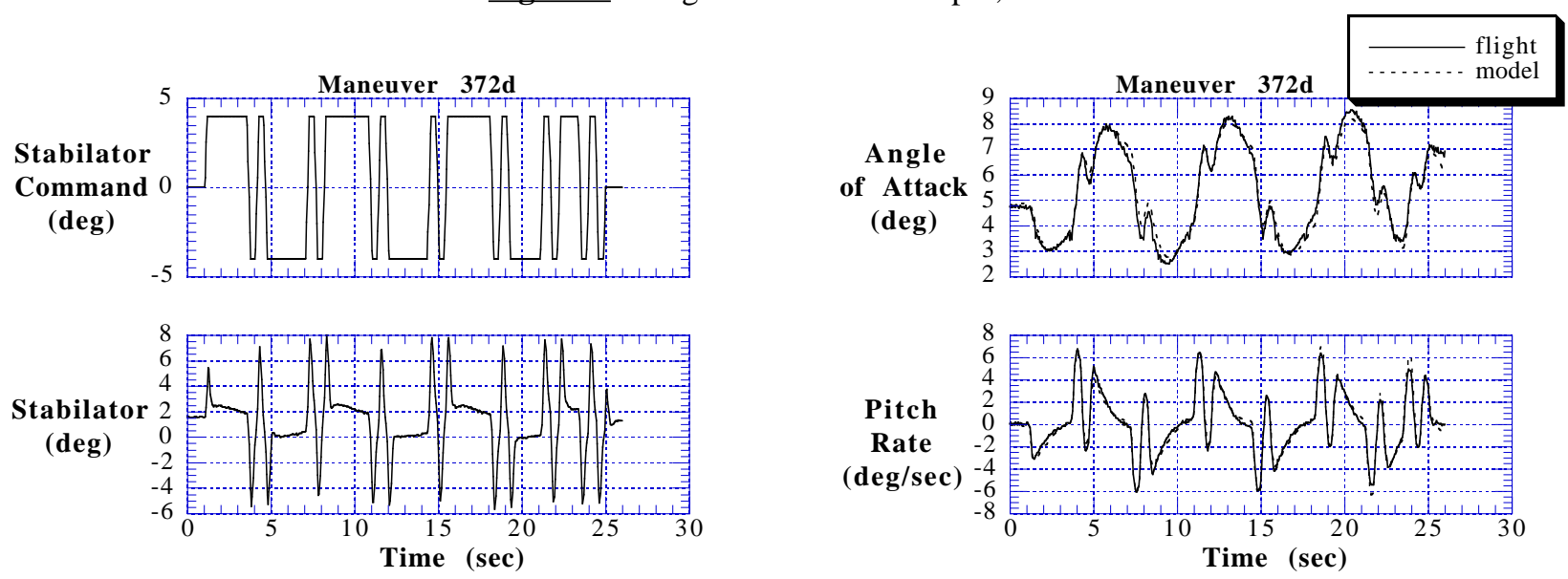

Figure 4 Longitudinal Optimal Input, $\alpha=5^{\circ}$
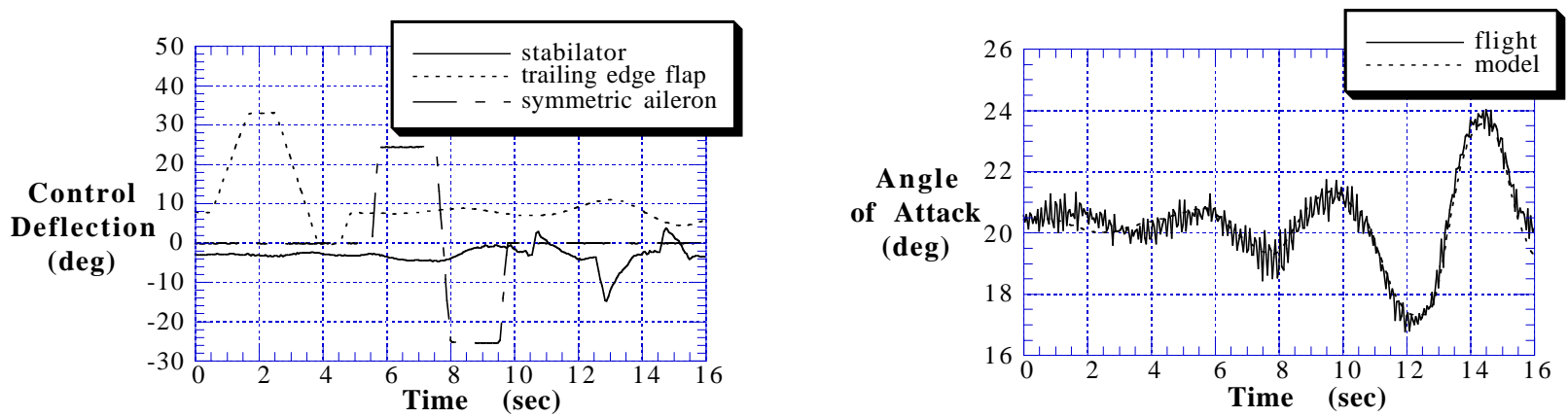

Figure 5 Longitudinal Doublet Input, $\alpha=20^{\circ}$
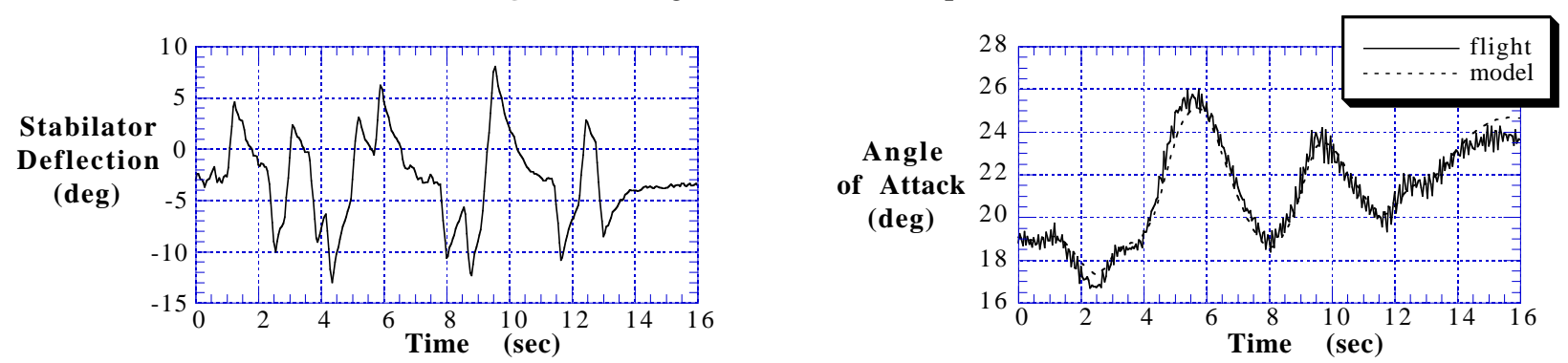

Figure 6 Longitudinal Optimal Input, $\alpha=20^{\circ}$ 
